\title{
Spatiotemporal underwater light field fluctuations in the open ocean
}

\author{
Martin Hieronymi \\ mhieronymi@ifm-geomar.de
}

Andreas Macke
Leibniz Institute of Marine Sciences IFM-GEOMAR, Kiel, Germany

Leibniz Institute of Marine Sciences IFM-GEOMAR, Kiel, Germany

The light availability in the upper layer of the open sea is subject to strong fluctuations due to focusing of surface waves. This paper shows measurements of downwelling spectral irradiances as well as spatiotemporal light field patterns along the water column. Results are interpreted with respect to diverse surface waves. Direct wind develops capillary and small gravity waves that affect the light regime only up to circa $5 \mathrm{~m}$ water depth. At high seas and below $5 \mathrm{~m}$ depth, light fluctuations can be described more accurately in terms of sea state parameters such as wave height and period, rather than wind speed. Between $3 \mathrm{~m}$ and $25 \mathrm{~m}$ water depth, waves with significant heights of $1.5 \mathrm{~m}$ to $2.5 \mathrm{~m}$ provoke the strongest intensity fluctuations. In general, fluctuation amplitudes decrease and periods extend with water depth where the coefficient of variation, $C V$, is in average four times higher above $2 \mathrm{~m}$ compared to $25 \mathrm{~m}$ water depth. [DOI: 10.2971/jeos.2010.10019s]

Keywords: light field fluctuations, underwater irradiance, water waves

\section{INTRODUCTION}

In many respects, solar irradiation into the upper ocean is of interest. Climatically relevant for example is the direct radiation supply and thus the warming of the sea water or indirect the impact on the carbon and oxygen cycle of the atmosphere via photosynthesis of phytoplankton. More than half of the integrated primary production occurs in depths less than $40 \mathrm{~m}$ [1] where light availability can be subjected to fluctuations. These fluctuations affect growth and photosynthesis of algae and planktonic organisms (for example, see [2]). Very short time steps (in the range of milliseconds) are required to capture photo-chemical reactivity in response to light variability [3]. Thus it is of interdisciplinary interest to better understand light regimes in the ocean in terms of fluctuation periods and ranges of intensity.

The underwater illumination and its fluctuations depend on the sun's position, the wavelength of light, cloud conditions, scattering properties of water and dissolved matter and of course the water depth. The main source mechanism of fluctuations in underwater irradiance is found to be the focusingdefocusing effect by surface waves [4]-[6]. Typically, highfrequency light flashes occur in the first meters of the water column. Dera and Stramski [7] defined light flashes as peak intensities exceeding the mean irradiance at a depth by a factor of 1.5 and in extreme cases more than 5 . With increasing water depth, fluctuation amplitudes in irradiance decrease because of light beam attenuation along the optical path and superposition of light focusing and defocusing regimes [5]. Strongest irradiance variations take place in shallow clear waters under direct sun at high altitudes and moderate wind conditions (with wind speeds between $1 \mathrm{~ms}^{-1}$ and $5 \mathrm{~ms}^{-1}$ ) [7]-[9].
The aim of this article is to improve the understanding of spatiotemporal variability of light regimes especially in the open ocean. Here, fully developed wave systems and thus larger gravity waves characterize light fluctuations in the water column. It is not necessarily the wind speed that defines the local water surface structure; on the high seas very often swells dominate the sea state (in terms of wave energy content) which comes along with superposed waves of even small gravity and capillary wave size that are responsible for high-frequency light flashes near the surface. In addition gravity waves act as larger lenses with deeper focal depths, which could be of interest for light utilization of phytoplankton below nutrient limited water layers.

To attain this goal we report measurements of spectral downwelling irradiance at the open sea. We introduce an underwater camera device for recording spatiotemporal light patterns along the water column. This tool gives additional information on fluctuation periods and spatial distribution of illumination.

\section{METHODS AND INSTRUMENTATION}

\subsection{In-situ measurements}

Measurements have been carried out in 2008 and 2009 on board RV POLARSTERN during three north-south traverses of the tropical and subtropical Atlantic Ocean (ANT 24/4, ANT 25/1, ANT 25/5; positions are marked in Figure 1). Within the framework of OCEANET the energy and material exchange between ocean and atmosphere is researched here. 


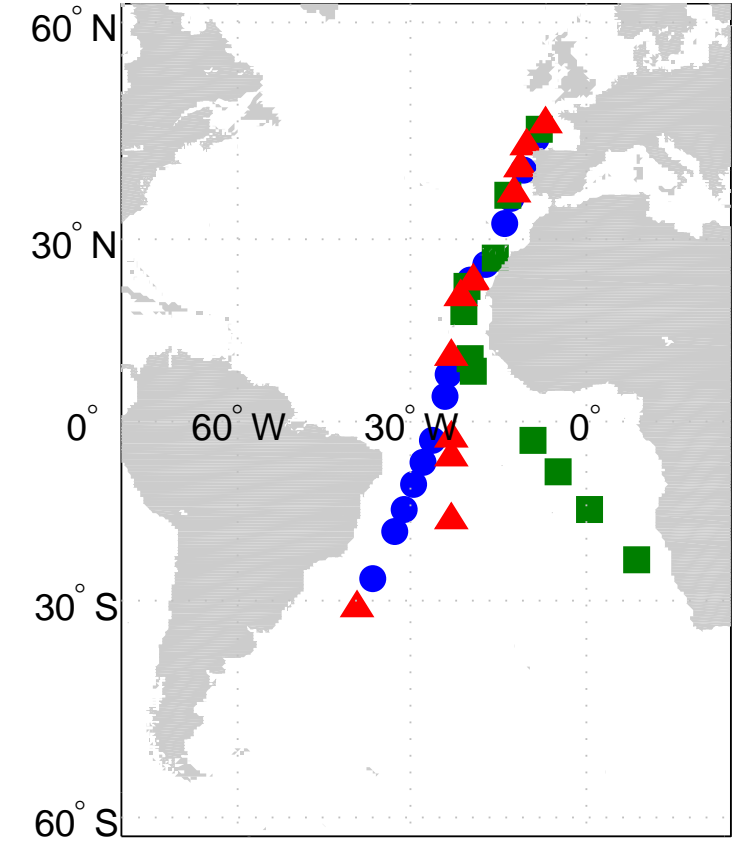

FIG. 1 Measuring sites on board the RV Polarstern at the Atlantic Ocean, circles: ANT24-4 (Apr-May 2008), squares: ANT25-1 (Nov 2008), triangles: ANT25-5 (Apr-May 2009).

Detailed datasets on atmospheric conditions (clouds, radiation budget, aerosols) and marine properties (water quality, optical characteristics, biomass concentrations and composition) are recorded.

Light fluctuations in the upper ocean are investigated under conditions that afford strong light variability, like direct sun, high sun altitude and not too choppy seas. These requirements limit the number of missions. Since the vessel is moving, some aspects need to be kept in mind, which may influence the results. The light attenuation depends on water composition, which was not the same at different stations. The water was sometimes turbid tropical-subtropical water and often extremely pure ocean water, but always case 1 water [10]. Additionally phytoplankton and thus scattering bodies were not distributed homogeneously in the water column. With changing ship positions, the maximum sun altitudes change as well. Our measurements were carried out at zenith angles always lower than $40^{\circ}$. However, according to Gernez and Antoine [9], the fluctuation parameters should be around the same order of magnitude at these zenith angles.

\subsection{Registration of sea state}

Sea state registrations are accomplished by onboard meteorologists via visual assessment and additionally by means of a wave radar system. The parameters distinguished for swell and wind-sea are the significant wave height (which is defined as the mean wave height of the one-third highest waves), the mean peak period and the propagation directions. Wind speed and direction are registered automatically.

Combined significant wave heights were between $1 \mathrm{~m}$ and $3.5 \mathrm{~m}$. In terms of the relative ratio of energy associated to each wave system, we had two of 37 times wind-sea dominated sea states, equally two cases with comparable influence of wind- sea and swell but mainly (33 of 37 times) swell dominant sea states [11]. Dominance of swell may imply strong sea surface deflections despite absence of wind.

\subsection{Spectral irradiance measurements}

In order to characterize the underwater light regime, radiometers are lowered from the working deck of the vessel over a crane arm. The vessel is orientated (according the sun's position and wave direction) in a way that minimizes influences of the ship's hull on radiation and waves fields. Still the shadow of the hull compromises the quality and the representability of the radiometric datasets [12]. This drawback and the draft of RV POLARSTERN of about $11 \mathrm{~m}$ should be kept in mind when interpreting the outcome. Other possible effects on the dataset result from ship motions that are transmitted to the tethered sensors. At a specific measurement depth, the displayed depth varies by up to $0.5 \mathrm{~m}$ due to these motions. Declinations of the radiometer are recorded but disregarded for the data analysis since the tilt angles are comparable small.

The sensor that is employed is a RAMSES-ACC-VIS. It maps downwelling irradiance in the UV and visible spectral range (320 nm-950 nm). Integration times for scanning the spectrum elongate with water depth, ranging typically in the upper $50 \mathrm{~m}$ from $8 \mathrm{~ms}$ to $512 \mathrm{~ms}$ but nevertheless, the sampling interval is around $2 \mathrm{~s}$. This sampling rate clearly is insufficient to capture high-frequency light flashes and even longer fluctuation periods can not be registered adequately. The mean value of irradiances can be better estimated when sampling over longer times at particular depths. The sensor package is lowered to certain depths (the vertical resolution changes with depth from $2 \mathrm{~m}, 2.5 \mathrm{~m}$ or $5 \mathrm{~m}$ steps up to a maximum depth of $50 \mathrm{~m}$ ) where it measures for $2 \mathrm{~min}$. With sixteen stops, a complete mission can last for $50 \mathrm{~min}$ with hopefully steady environmental conditions.

\subsection{Motion pictures of underwater light patterns}

Ship-based underwater light field measurements can be problematic as described above. Therefore, additional information that mark spatiotemporal variability of light intensity can be useful. Visualized spatial patterns changing with time allow straightforward conclusions on surface waves that cause certain light fields. Image analysis facilitates indication for light fluctuation amplitudes (strength of oscillations) and ranges of temporal fluctuation periods and spatial patterns respectively.

The system utilized consists of a commercial digital camera in an underwater housing which is mounted on a pyramidal frame with a white plate underfoot (technical specifications are given in Table 1). From above, the camera films the horizontal projection screen on which light patterns are displayed. This platform with tilt and pressure sensors is suitable down to maximal $40 \mathrm{~m}$ of water depth and fluctuations in brightness below $30 \mathrm{~m}$ depth are hardly detectable. The construction is lowered from a zodiac boat manually to compensate partly enormous hydrodynamic forces pulling at the screen due to wave motions. Water depth is to be seen as water col- 


\begin{tabular}{|c|c|}
\hline Camera & Canon PowerShot A620 \\
\hline resolution & 7.1 megapixels \\
\hline image size & 480 pixels $\times 640$ pixels \\
\hline image frequency & $30 \mathrm{fps}$ \\
\hline memory card & $2 \mathrm{~GB}$ \\
\hline Underwater housing & WP-DC 90 \\
\hline maximum depth & $40 \mathrm{~m}$ \\
\hline Projection screen & $0.4 \mathrm{~m} \times 0.6 \mathrm{~m}$ \\
\hline
\end{tabular}

TABLE 1 Specifications of the camera device.

umn above the plate and it is not feasible to hold the device in constant depths relative to the zero level with significant wave heights up to $3.5 \mathrm{~m}$. Another important advantage of operating from a zodiac is that the measurements can be taken from the undisturbed wave fields and away from light shadowing by the ship. Motion pictures of $1 \mathrm{~min}$ to $2 \mathrm{~min}$ length are taken at defined depths. The focus of the camera and the sensors integration time are held constant during measurements.

Limitations of the system can be due to, for example, shadows by the camera and by the frame on the projection screen (which happens often), sometimes inclination of the plate (distorted compared to a perfectly horizontal projection), automatic adjustments of the camera can not be avoided and motions in general must be accepted. In principle, one can steady the construction at depth; where the upward and downward transit often is related with movements and horizontal displacements which can lead to slack-cable situations that contain the risk of damage or lost. A grid with $10 \mathrm{~cm}$ spacing at the white plate allows areal mapping of the light fields and features bench marks for camera focusing.

\subsubsection{Image Analysis}

The first step of image analysis is to cut the film into single pictures and allocate those to the pertinent depths. Images are composed of red, green and blue pixel information (RGB value) which assigns the spectral intensity. In the first meters of the water column pixel values of each colour develop temporally similar with high correlation. With increasing water depth the portions of colour separate. Green and blue parts are still quite similar and well-correlated. The red information disappears because of stronger absorption in this spectral band. Below $10 \mathrm{~m}$, the images are noticeable bluish and greenish in more turbid water. Analyses in the time and frequency domain are performed separately for each colour portion. In the following the so called "gray value" is used. This value mirrors the pixel brightness independently of the spectral range. The colours are averaged weighted by the spectral sensitivity of the human eye.

$$
\mathcal{G}=0.299 R+0.587 G+0.114 B
$$

where $\mathcal{G}, R, G$ and $B$ represent the gray, red, green and blue values respectively.

\subsubsection{Time domain analysis}

Only image pixels are considered that are not subjected to shadows or other disturbances like air bubbles. Each time se- ries has a length of one minute with $30 \mathrm{~Hz}$ resolution. One parameter used to characterize light intensity fluctuations is the amplitude, which is quantified by the coefficient of variation, $C V$, is given by [9]

$$
C V=100 \frac{\sigma_{m}}{\mu}
$$

where $\sigma_{M}$ is the standard deviation relative to the median and $\mu$ is the median of the time series. Figure 2 shows a typical time series of a pixel gray value at $10 \mathrm{~m}$ depth. The median and standard deviation are indicated by solid lines.

Note that the statistics of the image pixel intensity fluctuations cannot be related directly to that of the downwelling irradiance. Especially in the upper meters, time series of irradiance typically show extreme enhancements (light flashes). Thus the irradiance distribution is skewed [7]. Compared to this, the spatiotemporal distributions of pixel brightness are more Gaussian. Extreme peaks are bounded above by the upper limit of RGB values (colour combination leads to a white pixel). A second reason for not recording extreme light flashes (and thus normal distribution of light intensity) is the camera's sensitivity and internal image processing. Nevertheless, for all intents and purposes the coefficient of variation can be qualitatively related to fluctuation amplitudes. $C V$ is of around the same magnitude comparing the gray level with the irradiance at a depth.

\subsubsection{Frequency domain analysis}

The frequency band or the mean period is the second parameter to specify fluctuations. The temporal change of a pixel's brightness is evaluated by means of its spectral density (also variance spectrum). Based on fast Fourier transform (FFT) and Welch's method, as it is implemented in MATLAB, the frequency content of the signal is estimated. In Figure 2, a typical variance spectrum of corresponding time signal is shown. The maximum of the frequency weighted spectrum indicates the averaged peak period, $T_{P}$, of the signal, which often can be seen directly as a major superposed oscillation in the time signal (in the shown example the dominant period is around $1 \mathrm{~s})$. This parameter is not always suitable and can lead to inaccuracy especially at wide spectral maxima or peaks in close neighbourhood. That problem can be faced by the application of spectral moments given by

$$
m_{i}=\int_{0}^{\infty} f^{i} S(f) d f
$$

where $f$ is the frequency and $S$ is the spectral density of the signal. The mean period of fluctuation indicates the spectral centre of gravity and thus weighs the relevant frequency band more,

$$
T_{m}=\frac{m_{0}}{m_{1}}
$$

i.e. the area below the integral divided by the first moment. The mean period can be seen as grade of light field smearing. Normally, the mean period, $T_{M}$, is smaller than $T_{P}$ and differences increase with depth. Both parameters are adequate to specify the signal's fluctuation. 

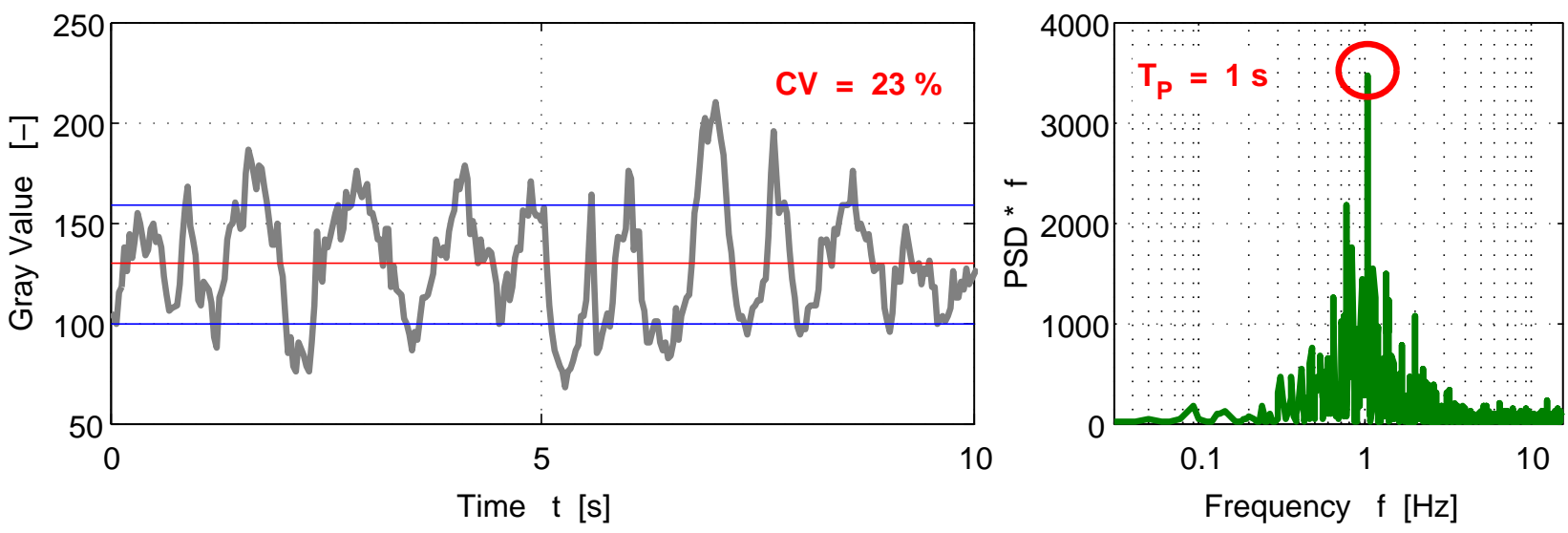

FIG. 2 (Left) Typical time series of a pixel gray value at $10 \mathrm{~m}$ depth (the red line shows the median while the blue lines indicate the standard deviation). (Right) Frequency weighted power spectral density (PDS) for the same time series.

\subsubsection{Spatial light fields}

Images provide areal information on the grade of attenuation and overlapping of clustered light beams. Within the first meters, the major portion of visible light is not absorbed yet; which leads to potentially strong superposition due to the lensing effect. Depending on the electromagnetic wavelength, light is refracted at the air-water interface with different focal depths. For example, bluish light emerges at deeper focal points than red light for a given lens profile. And because of stronger absorption in the reddish frequency band, blue light develops more extreme intensity when focusing. Thus a horizontal plane contains different development of colourdepending focal planes.

Small scale structures of the mirrored water surface can be identified at pictures taken from $1 \mathrm{~m}$ of water depth (see Figure 3). The distance between two local intensity maxima at a line shows the wavelength of the causing surface waves. Smallest distances correspond to approximately $1 \mathrm{~cm}(0.01 \mathrm{~m})$ long capillary waves, whose dynamics are dominated by the effects of surface tension. The most intense brightness variations in $1 \mathrm{~m}$ depth result from $20 \mathrm{~cm}$ to $35 \mathrm{~cm}$ long waves, which are small gravity waves. Information on wave heights is lost due to the $3 \mathrm{D}$ projection of the deflected surface on a $2 \mathrm{D}$ plane.

At $5 \mathrm{~m}$ water depth, spatial light fields are more diffuse. Smallest distinguishable structures are around $10 \mathrm{~cm}$ in distance. The patterns are dominated by intensity peaks of more than $25 \mathrm{~cm}$ space. Figure 4 illustrates typical temporal changes of image cuts at $1 \mathrm{~m}$ and $5 \mathrm{~m}$ depth. Small structures along image profiles smear with depth, but the magnitudes of intensities can increase in a middle range of depth (from $2 \mathrm{~m}$ to $7 \mathrm{~m}$ ) which is indicated by the colour bar. The smear of the patterns is more obvious in Figure 5, where the spatial correlation is plotted against pixel distance (mean values and standard deviations for $1 \mathrm{~m}, 6 \mathrm{~m}$ and $20 \mathrm{~m}$ ). While in the upper meters the correlation decreases fast after some millimetres of pixel distance, correlation is high at larger depths. In $20 \mathrm{~m}$ depth, the whole picture is more or less of the same colour that brightens and darkens slowly with time.

FFT based spatial wavenumber analysis is inapplicable

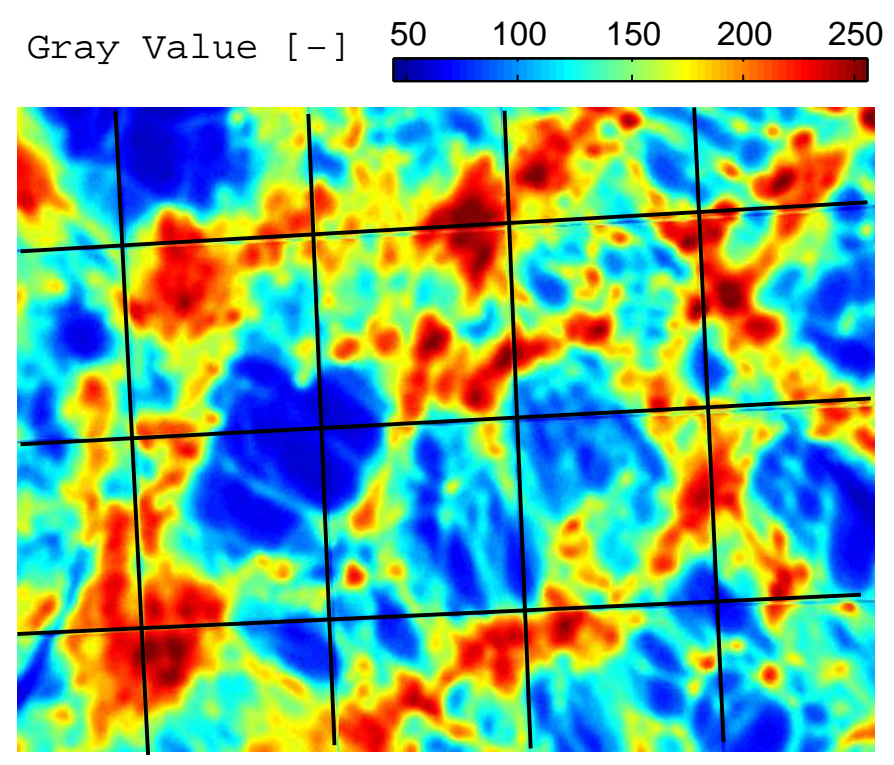

FIC. 3 Snapshot of a typical light field pattern at $1 \mathrm{~m}$ depth (colour-indicated gray values). Shades of the frame and the camera may occur. The highlighted grid indicates $10 \mathrm{~cm}$ ranges.

due to the limited size of the image plane (approximately $35 \mathrm{~cm} \times 45 \mathrm{~cm}$ ). Instead of the time series of a single-pixel brightness, only a directed one-dimensional image cut of $45 \mathrm{~cm}$ length can be used. With dominant light patterns with spatial distances of more than $23 \mathrm{~cm}$ between intensity maxima, this approach lacks accuracy. Thus the wavelength as a descriptive fluctuation parameter is less reasonable for the given experimental setup.

For gravity waves, spatial information can be gained indirectly out of dispersion relation of the surface wave period and the wavelength. In linear wave theory, the wavelength (for deep water) is

$$
L=\frac{g}{2 \pi} T^{2}
$$

where $g$ is the acceleration of gravity and $T$ is the wave period. In this context, wavelength describes the distance between two peaks (or local maxima) of light intensity. 


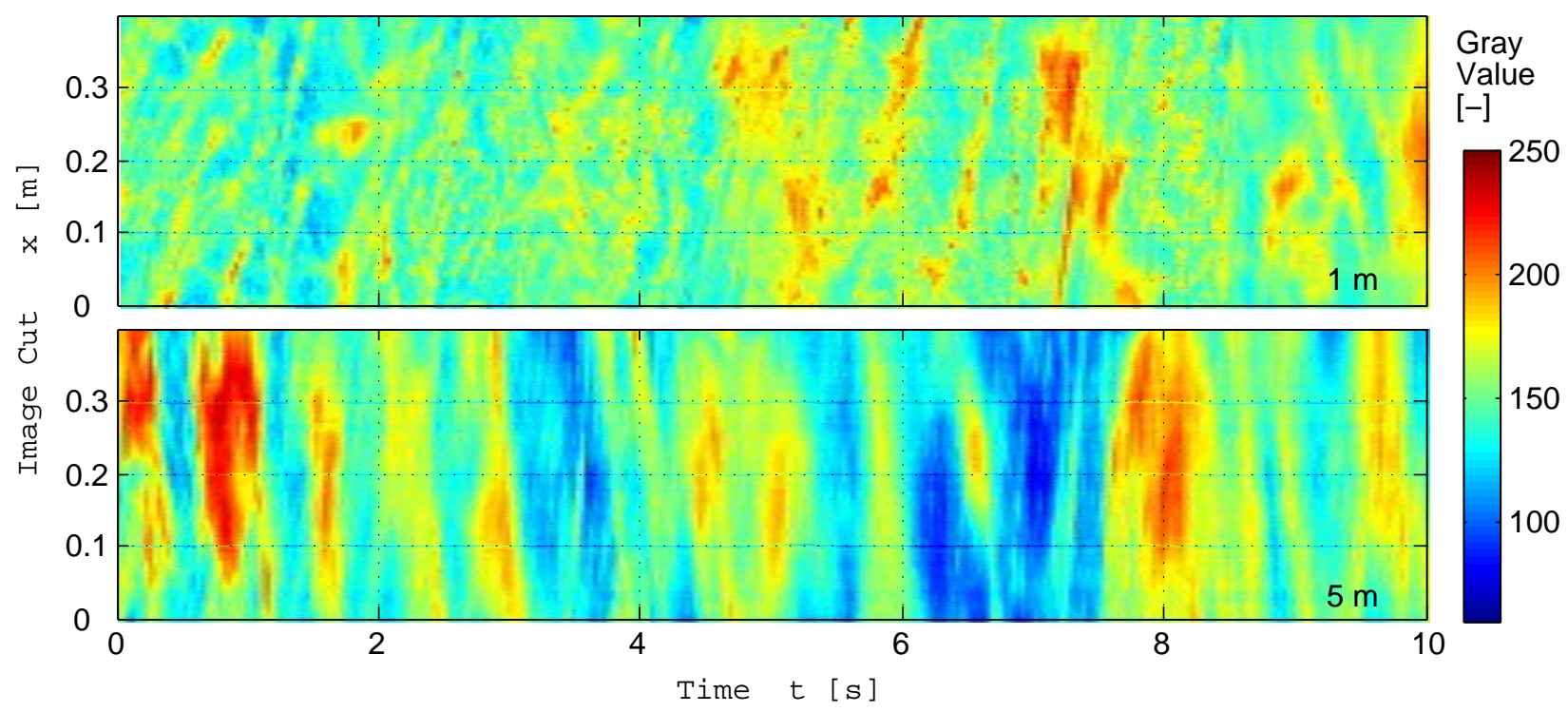

FIG. 4 Spatiotemporal light fields in $1 \mathrm{~m}$ and $5 \mathrm{~m}$ depth $-40 \mathrm{~cm}$ spatial gray value structure versus its temporal change.

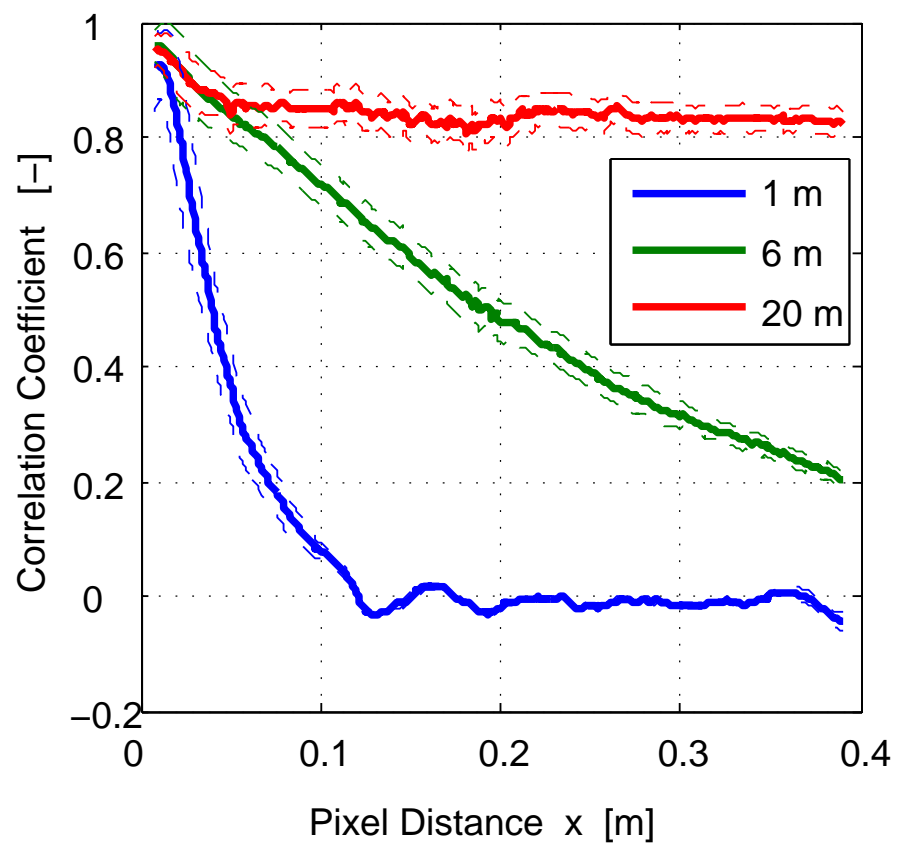

FIC. 5 Typical correlation of temporal changes of the image pixel intensity with respect to the distance of image pixels for three depths (solid lines represent the mean values and the dashed lines correspond to the standard deviation at the particular depths.

\section{RESULTS AND DISCUSSION}

\subsection{Irradiance measurements}

By collecting 2 min data per depth level (about 60 spectra), we obtain a broader view of the light supply and variance in the water column. As an example, Figure 6 shows spectrally resolved irradiance measurements in $10 \mathrm{~m}$ and $25 \mathrm{~m}$ depths. The mean spectra are plotted in red while minima and maxima are denoted by the gray-shaded area to show wavelengthdepending ranges of fluctuations. The visible frequency band is additionally illustrated by a corresponding spectral colour scale. Most intensity is associated with wavelengths between $400 \mathrm{~nm}$ to $575 \mathrm{~nm}$ (blue and green range). At every depth, the peak of the irradiance spectra is at wavelengths around

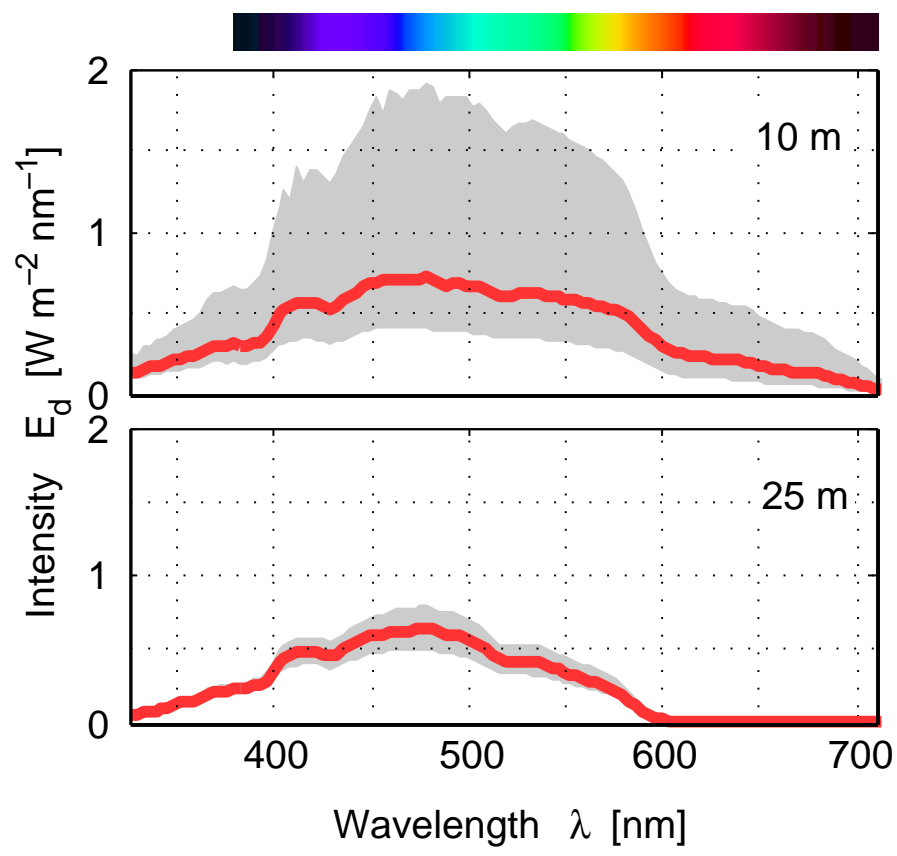

FIG. 6 Spectral downwelling irradiance measurements in $10 \mathrm{~m}$ and $25 \mathrm{~m}$ depths. The red lines show mean spectra, shadowed area show ranges of measured intensities with minimal and maximal spectra, the colour bar at the top illustrates the visible colour spectrum corresponding with the wavelengths below.

$480 \mathrm{~nm}$. In $10 \mathrm{~m}$ depth, the maximum spectrum is, depending on wavelength, more than 2.5 times of the mean irradiance $(>2.7 \bar{E})$, while lowest intensity is less than half of mean value $(<0.5 \bar{E})$. In $25 \mathrm{~m}$, the irradiance in the red band is absorbed almost fully. At $480 \mathrm{~nm}$, the maximal measured irradiance is round $1.3 \bar{E}$ and minimum less than $0.7 \bar{E}$.

Now we focus on a single wavelength from the frequency band that potentially penetrates deepest into the water. Figure 7 shows the measured downwelling irradiance against depth at the particular wavelength $462 \mathrm{~nm}$ relative to the value that was measured above the water surface. The $100 \%$ line of the surface value is highlighted. All dots mark the monochromatic data and squares indicate their mean values 


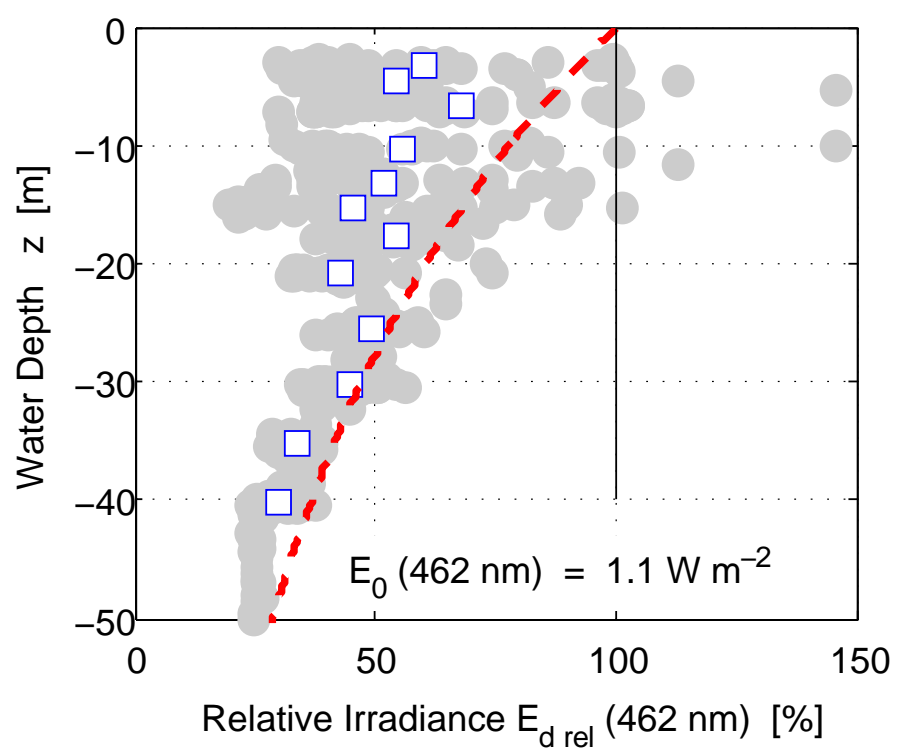

FIG. 7 Irradiance measurements of bluish light ( $462 \mathrm{~nm}$ ) with water depth relative to the intensity at the sea surface. Squares show the mean values for the data within $\pm 0.5 \mathrm{~m}$

for a horizontal layer within $\pm 0.5 \mathrm{~m}$ (that is due to hydrodynamic motions). The downwelling irradiance $E_{d}(\lambda)$, which we measure with a horizontally orientated cosine collector, describes the flux per unit area. The propagation of light in clear and calm water is comparatively monodirectional. Thus it can be useful for comparison to treat the irradiance $E_{d}(\lambda)$ as proportional to the light intensity $I(\lambda)$. The dashed line in Figure 7 stands for the theoretical intensity attenuation in pure water with calm surface,

$$
I(\lambda, z)=I_{0, \lambda} \exp \left(-k_{\lambda} z\right)
$$

where $I_{0, \lambda}$ is the intensity at the air-water interface, $k_{\lambda}$ is the extinction coefficient of water at the particular wavelength $\left(k_{462 \mathrm{~nm}}=0.025 \mathrm{~m}^{-1}\right.$ interpolated from [13]) and $z$ is the optical path. The attenuation coefficient considers absorption and scattering.

The first thing to note is that the shadow of the vessel (from which the device is lowered) may affect the results at the left hand side of the diagram with lower values. When lowered, the vessel's shadow appears in the field of view of the sensor, which is influenced by Snell's circle [12]. Due to scattering processes, the shadow plays a minor role once the device is deep enough. The predominant direction of light rays in clearest water is from a relative small circle directly above. So the shadow may have less impact on data. Another explanation for extreme small values above $20 \mathrm{~m}$ depth and for the clear dent of minimal values around $25 \mathrm{~m}$ is the focusing effect and a region of focal planes that depend on surface wave types. At the focal plane of a single wave, below the wave's crest an extreme peak appears, but in the close neighbourhood to the peak, very low intensity values must be registered at the same depth level. The way to proof this idea would be to perform high-frequency measurements with reasonable vertical resolution.

Extreme large irradiance values can reach more than $150 \%$ of the surface insolation even at $10 \mathrm{~m}$ depth. This, as described above, corresponds to nearly three times the averaged value at that depth. The irradiance averaging per depth must be taken with care. Ideally the mean intensity must decrease exponentially with depth as shown by the dashed line. Since the sampling rate is not sufficient, only a few of the short-term values of very large intensities can be recorded. This leads to an underestimation of the light intensity in the first tens of meters. This by the way is a problem of all standard procedures of light profiling. A typical feature of such profiles that should be appointed is the transformation of the intensity distributions with depths from strong asymmetric and skewed distributions in the upper meters to more normal distributions below $10 \mathrm{~m}$ depth. In light foci much more intensity is cumulated than somewhere else at the focal plane and extreme low intensities of the same magnitude do not occur. Once light fields are widened and became blurred the irradiance time series are more harmonically and thus normally distributed.

\subsection{Temporal light field fluctuations}

The movie analysis results in parameter describing the strength of fluctuation amplitudes using the coefficient of variation, $\mathrm{CV}$, and a time component, the mean fluctuation period, $T_{M}$. These values can be related to environmental conditions as sea state (period and wave height of both swell and wind sea which is obtained by visual assessment), wind speed, water properties (turbidity, Chlorophyll-a stratification), sun altitude and water depth. Because of the limited number of data, just indications on those relationships can be given. Water properties and sun positions are not considered. Interesting clues on fluctuation influences can be found when interpreting $C V$ and $T_{M}$ in terms of wind speed (which is comparable with other publications, see $[7,9]$ ) and significant wave height as a combined parameter from the mixed sea. Accordingly in Figure 8, the coefficients of variation and the mean fluctuation periods are compared and plotted versus water depth. In previous works, mostly light regimes in two to four water depths are investigated, this study presents data from eleven depths and thus gives a more detailed vertical resolution. Every dot stands for one analysed movie at a certain depth. Wind speed and wave height are examined separately because of swell dominance of sea states, as explained before. In principle, wave height grows with the speed of wind, which can be seen in the datasets.

With regards to the $C V$, the strongest fluctuations appear at relative low wind speeds $\left(<6 \mathrm{~ms}^{-1}\right)$ within the first two meters, which is consistent with [7,9]. Higher wind speeds increase the mean square slope of the sea surface regardless of wind direction [14]. This impairs the efficiency of generating lens-surfaces for intense focusing; that is the $C V$ decreases. Local winds provoke the development of small-size waves. Their impact on the light regimes in terms of the focusing effect is limited to the upper meters of the water column (roughly $5 \mathrm{~m}$ in clear waters). From this perspective, $\mathrm{CV}$ seems to lose its wind dependency with increasing water depth. At $20 \mathrm{~m}$ depth, largest fluctuations can be observed at relatively strong winds (around $7 \mathrm{~ms}^{-1}$ ). Here, light fields disperse and depend more on large scale structures of the water surface shape. The parameter wind speed is less attractive for describing the nature of the light field in terms of intensity amplitude 


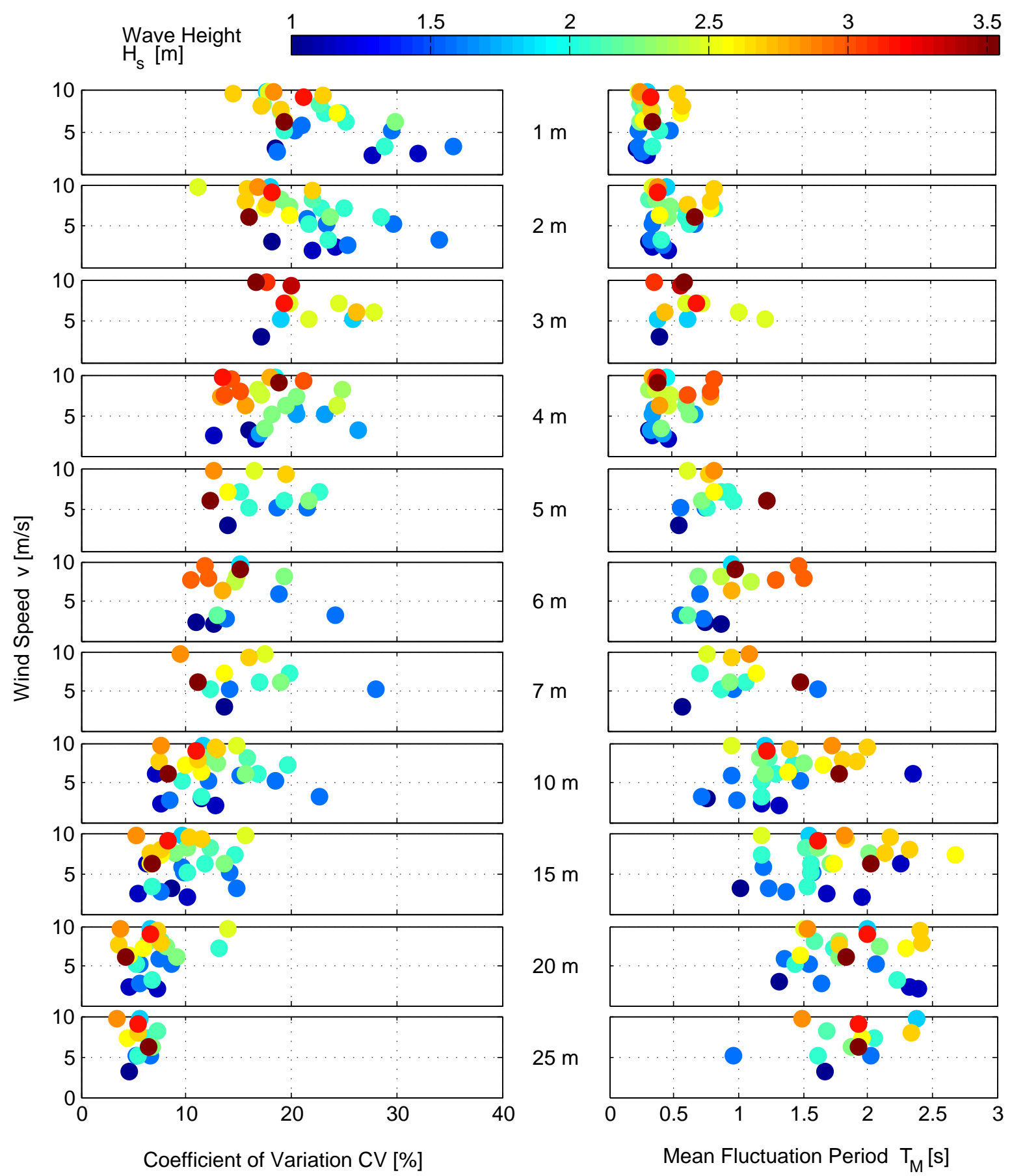

FIG. 8 Results of the image analysis: coefficient of variation (left) and mean fluctuation period (right) with depth depending on speed of wind ( $y$-axis) and significant wave height (color-indicated).

variations. In this case, the wave height (which is correlated with wind speed and a term called wave-age) is more advisable. Between $3 \mathrm{~m}$ and $20 \mathrm{~m}$ depth, significant wave heights of around $1.5 \mathrm{~m}$ to $2.5 \mathrm{~m}$ cause major fluctuations. Hypothetical higher and larger waves control strength of intensity fluctuations in even deeper waters. In general, fluctuation amplitudes decrease with depth.

With regards to $T_{M}$, the mean fluctuation periods increase with depth from high-frequency dominance below $2 \mathrm{~Hz}(<$ $0.5 \mathrm{~s})$ to slow varying of up to $3 \mathrm{~s}$ which comes along with dominant and clear peak periods of up to $6 \mathrm{~s}$. The data show a tendency towards longer light fluctuation periods when the wind speed grows. This is equivalent to rising waves with longer wave periods. Below $10 \mathrm{~m}$, mean and peak periods signify distances (wavelengths) of light intensity maxima of $2 \mathrm{~m}$ to $40 \mathrm{~m}$. This gives evidence of gravity wave and sea state impact on underwater light regime. Even swell waves with periods of $5 \mathrm{~s}$ to $10 \mathrm{~s}$ (wavelengths of $40 \mathrm{~m}$ to $150 \mathrm{~m}$ ) provoke potentially significant fluctuations of light intensity in deeper waters.

\section{SUMMARY AND CONCLUSIONS}

The focusing effect of light due to lenses formation of surface waves is the main reason for light intensity fluctuations in the water column. This work surveys the impact of surface deformations on light regimes at open ocean conditions. 
Measurements of spectral downwelling irradiance in the upper $50 \mathrm{~m}$ of the water column are presented and interpreted with respect to the focusing effect. Furthermore, an off-theshelf underwater camera mounted on a projection plate is deployed for spatiotemporal light field visualization.

In principle, cumulated light beams can penetrate deeper and can deliver punctual higher intensity into water. The shape of the water surface specifies the light intensity distribution in the water column.

Small gravity and capillary waves cause high-frequency (> $1 \mathrm{~Hz}$ ) intensity fluctuations with focal depths in relative shallow water (roughly above $5 \mathrm{~m}$ ). With adequate sampling rates, extreme intensity peaks can be detected (see [7]). Free-falling irradiance measurements and sampling-per-depth profiling with low sampling rate lead to an underestimation of mean intensity in the water column. Here, the camera system supports data interpretation. In the first meter light fields are well pronounced. They show mirroring of capillary waves patterns with lengths as small as $1 \mathrm{~cm}$; the most intense brightness variation result form $20 \mathrm{~cm}$ to $35 \mathrm{~cm}$ long surface structures, which matches small gravity waves. The speed of wind is correlated to fluctuation amplitudes in terms of the coefficient of variation. Relative slow winds $\left(2 \mathrm{~ms}^{-1}\right.$ to $\left.7 \mathrm{~ms}^{-1}\right)$ cause strongest fluctuations and the $C V$ tends to decrease with increasing wind speed (which is a confirmation of previous works, see for example [9]).

With depth, light fields smear because of scattering and overlaying of diverse developed focal depth. The light regime in between $3 \mathrm{~m}$ to $25 \mathrm{~m}$ of water column is affected by large scale surface structures. Significant wave heights of $1.5 \mathrm{~m}$ to $2.5 \mathrm{~m}$ provoke the strongest intensity oscillations and the speed of wind is only indirectly correlated via fully developed wind sea. When swells or premature wind seas dominate the sea state, which is often the case with high seas, the wind speed is no suitable comparative parameter. Ordinary gravity waves specify the irradiance distribution in this section of water column. Mean periods of intensity fluctuations range up to $3 \mathrm{~s}$, which corresponds to outstanding averaged peak periods of up to $6 \mathrm{~s}$. Dispersion relation shows light filed structures of $2 \mathrm{~m}$ to $40 \mathrm{~m}$ wavelength below $10 \mathrm{~m}$ water depth. With depth, brightness oscillations go decelerated up and down with smaller amplitudes.

Even swell waves with periods of $5 \mathrm{~s}$ to $10 \mathrm{~s}$ (which is equivalent to wave lengths between $40 \mathrm{~m}$ to $150 \mathrm{~m}$ ) have potential to provoke significant fluctuations of light intensity in deeper waters. This is of relevance for light availability in the major part of the euphotic zone and thus of can be of interest for all photo-relevant processes.

The data basis of light regimes at different sea state scenarios is limited and up to now not sufficient for a strict argumentation. It is shown in this work that gravity waves and also swell influence the light availability at high sea. Long surface waves enable light to potentially penetrate deeper into water which is of high interest for phytoplankton and biogeochemical processes. Radiative transfer simulations by means of the Monte
Carlo method with diverse ocean wave scenarios could shed light onto this matter.

\section{ACKNOWLEDGEMENTS}

Martin Hieronymi acknowledges the financial support of the Kiel Cluster of Excellence FUTURE OCEAN. The work results from cooperation within the OCEANET framework; amongst others the phyto-optics group at AWI and IUP University of Bremen with Astrid Bracher, Bettina Schmitt, Anja Theis and Laila Bentama. The measurements have been carried out together with members of the working group in Kiel, namely Yann Zoll, Timo Hanschmann, John Kalisch, Karl Bumke, Katharina Lengfeld, Michael Schlundt and Andreas Wassmann. The support of the RV POLARSTERN crew (ANT 24/4, ANT 25/1 and ANT 25-5) is highly appreciated.

\section{References}

[1] D. A. Siegel, A. F. Michaels, J. C. Sorensen, M. C. O'Brien, and M. A. Hammer, "Seasonal variability of light availability and utilization in the Sargasso Sea" J. Geophys. Res. 100, 8695-8713 (1995).

[2] R. M. Greene, and V. A. Gerard, "Effects of high-frequency light fluctuations on growth and photoacclimation of the red alga Chondrus crispus" Mar. Biol. 105, 337-344 (1990).

[3] 0. C. Zafiriou, J. Joussot-Dubien, R. G. Zepp, and R. G. Zika, “Photochemistry of natural waters" Environ. Sci. Technol. 18, 358A-371A (1984).

[4] H. Schenck, "On the focusing of sunlight by ocean waves" J. Opt. Soc. Am. 47, 653-657 (1957).

[5] R. L. Snyder, and J. Dera, "Wave-induced light-filed fluctuations in the sea" J. Opt. Soc. Am. 60, 1072-1079 (1970).

[6] D. Stramski, and J. Dera, "On the mechanism for producing flashing light under a wind-disturbed water surface" Oceanologia 25, 5-21 (1988).

[7] J. Dera, and D. Stramski, "Maximum effects of sunlight focusing under a wind-disturbed sea surface" Oceanologia 23, 15-42 (1986).

[8] M. Stramska, and T. D. Dickey, "Short-term variability of the underwater light field in the oligotrophic ocean in response to surface waves and clouds" Deep-Sea Res. Pt. I 45, 1393-1410 (1998).

[9] P. Gernez, and D. Antoine, "Field characterization of wave-induced underwater light field fluctuations" J. Geophys. Res. 114, C06025 (2009).

[10] A. Morel, and L. Prieur, "Analysis of variations in ocean color" Limnol. Oceanogr. 22, 709-722 (1977).

[11] G. R. Rodriguez, and C. Guedes Soares, "Correlation between successive wave heights and periods in mixed sea states" ocean Eng. 28, 1009-1030 (2001).

[12] R. C. Smith, "Structure of solar radiation in the upper layers of the sea" in Optical Aspects of Oceanography, N. G. Jerlov, and E. Steeman Nielsen, eds., 95-119 (Academic Press, New York, 1974).

[13] G. M. Hale, and M. R. Querry, "Optical constants of water in the $200 \mathrm{~nm}$ to $200 \mu \mathrm{m}$ wavelength region" Appl. 0pt. 12, 555-563 (1973).

[14] C. Cox, and W. Munk, "Measurements of the roughness of the sea surface from photographs of the sun's glitter" J. Opt. Soc. Am. 44, 838-850 (1954). 